\title{
Le métier de chef de projet comme activité prudentielle.
}

Enquête dans un groupe industriel du secteur de l'environnement

Project management and prudential activity. A survey in an industrial group of

the environmental sector

\section{Mathieu Bensoussan et Rémi Barbier}

\section{OpenEdition}

\section{Journals}

Édition électronique

URL : http://journals.openedition.org/travailemploi/5986

DOI : $10.4000 /$ travailemploi.5986

ISSN : 1775-416X

\section{Éditeur}

DARES - Ministère du Travail

\section{Édition imprimée}

Date de publication : 15 juin 2013

Pagination : 41-58

ISSN : 0224-4365

\section{Référence électronique}

Mathieu Bensoussan et Rémi Barbier « Le métier de chef de projet comme activité prudentielle. », Travail et Emploi [En ligne], 134 | avril-juin 2013, mis en ligne le 01 avril 2015, consulté le 19 avril 2019 URL : http://journals.openedition.org/travailemploi/5986 ; DOI : 10.4000/travailemploi.5986 


\title{
Le métier de chef de projet comme activité prudentielle. Enquête dans un groupe industriel du secteur de l'environnement $\left.{ }^{*}\right)$
}

\author{
Mathieu Bensoussan ${ }^{(* *)}$ Rémi Barbier ${ }^{(* *)}$
}

Si la gestion de projet est une activité désormais répandue dans les entreprises, le contenu du travail qu'elle implique reste paradoxalement mal connu. En s'appuyant sur une enquête menée auprès de chefs de projet d'un groupe industriel et sur les catégories de l'activité "prudentielle» proposées par Florent Champy, cet article rend intelligible ce contenu. Il montre que le chef de projet adopte un regard global sur son projet, surveille les contingences pouvant nuire à son déroulement et mobilise un "sens du possible» pour résoudre les problèmes auxquels il est confronté et arbitrer entre des exigences contradictoires. Le lien de subordination inhérent à son statut de salarié contraint son autonomie, mais lui procure aussi un certain nombre d'appuis organisationnels confortant la construction de sa vision globale du projet, de sa vigilance et de son sens du possible : la prudence apparaît sous la forme d'une qualité distribuée dans un réseau étroit de dispositifs organisationnels.

La majorité des travaux académiques qui portent sur la gestion de projet ${ }^{(1)}$ interrogent les effets de la «logique projet» sur l'organisation du travail dans les entreprises. Par contre, le contenu du travail de chef projet y est peu abordé. Généralement, le rôle formel du chef de projet est décrit, sa position dans l'organisation de son entreprise est analysée et la dimension comportementale de sa compétence (son leadership) est soulignée. On sait ainsi que le travail d'un chef de projet consiste formellement à «structurer méthodiquement et progressivement une réalité à venir»(2). Le chef de projet mobilise une myriade d'acteurs du travail, sur lesquels il n'a pas de pouvoir hiérarchique, mais dont il coordonne l'activité et entretient la coopération en vue d'atteindre un but déterminé (dans un délai et avec un impératif financier arrêtés ex ante). Sa réussite dépend de sa faculté à obtenir de ces acteurs qu'ils

(*) Cette étude a été réalisée dans le cadre de la chaire d'entreprise Suez-Environnement - ENGEES en «Management de Projet». Nous remercions les personnes ayant participé à l'enquête. Nous remercions également Isabelle Mauz et Didier Demazière, qui ont discuté une version antérieure du texte lors des «Journées des sociologues» de l'Institut de recherche en sciences et technologies pour l'environnement et l'agriculture (Irstea), le 7 octobre 2011. Nous remercions enfin les lecteurs de Travail et emploi pour leurs commentaires et suggestions qui ont grandement contribué à améliorer l'article.

(**) UMR Gestion territoriale de l'eau et de l'environnement (Geste), École nationale du génie de l'eau et de l'environnement de Strasbourg (ENGEES); mathieu.bensoussan@wanadoo.fr (***) UMR Gestion territoriale de l'eau et de l'environnement (Geste), École nationale du génie de l'eau et de l'environnement de Strasbourg (ENGEES); remi.barbier@engees.unistra.fr

(1) Les expressions «management de projet» et «gestion de projet» sont synonymes dans cet article.

(2) Selon la définition de la gestion de projet donnée par l'Agence française de normalisation (Afnor). agissent d'une manière congruente à la finalité d'ensemble (GAREL, 2011 (3)).

Pour l'essentiel, la gestion de projet reste une affaire d'ingénieurs diplômés (KARVAR, 2004) ${ }^{(4)}$. D'ailleurs, les projets les plus fréquemment étudiés par les sociologues et les chercheurs en gestion sont des projets d'innovation industrielle, et les chefs de projet dont il est question dans leurs écrits sont toujours des ingénieurs. Les sociologues du travail indiquent que l'organisation par projet, dans l'industrie, a transformé le travail des ingénieurs chargés de conduire l'innovation (Bouffartigue, 2001). Car, désormais dominé par la construction et la gestion de liens coopératifs dans les situations de travail (VINCK, 1999), ce travail réclame des compétences qui sont loin d'être strictement techniques. La gestion de projet peut donc être vue comme une espèce du travail d'encadrement : un travail visant «à trouver des compromis productifs, à ajuster en permanence des politiques, des objectifs, des indi-

(3) L'ouvrage de Gilles Garel est un ouvrage de synthèse, qui inventorie les connaissances produites sur la gestion de projet par les chercheurs en science de gestion. Sa visée étant opérationnelle (comment «piloter un projet», «constituer» et «animer» une «équipe projet», etc.), nous l'utilisons surtout comme une source documentaire.

(4) Une association professionnelle des chefs de projet existe depuis 1982: 1'Association française des ingénieurs et techniciens d'estimation et de planification (Afitep). Bien qu'elle soit dorénavant nommée «Association francophone du management de projet», ses statuts précisent que ses «membres actifs » sont des «ingénieurs » et des «techniciens » (article 3 ). L'Afitep revendique un millier d'adhérents physiques. Elle participe activement au travail de normalisation en management de projet qui a été engagé en 2000 (sur son initiative) par l'Afnor et certifie aussi les compétences des chefs de projet. 
vidus et des moyens, à fabriquer de la cohérence locale», et qui renferme une «dimension comportementale forte» (Livian, 2004, p. 6).

L'article prolonge et élargit la perspective, en mettant l'accent sur les contenus de travail par lesquels la «structuration d'une réalité à venir» s'opère. Il s'appuie sur une enquête conduite en 2010-2011 dans trois entreprises, qui sont trois filiales d'un groupe industriel du secteur de l'environnement. La première d'entre elles gère les déchets (municipaux ou industriels) et sera nommée «société de déchets». La seconde, que nous appellerons «société exploitante»- elle exploite des usines -, produit de l'eau potable et assainit les eaux usées par délégation des collectivités publiques. Quant à la troisième, elle conçoit et construit des usines de production d'eau potable et des usines d'assainissement des eaux usées (elle sera désignée «entreprise de construction») ${ }^{(5)}$. Des projets sont conduits dans ces trois entreprises. Leur signification est spécifique à chacune d'entre elles ${ }^{(6)}$ (voir encadré 1).

Les projets sont donc ici de deux types On trouve ainsi les projets qui consistent à produire et à remettre un dossier (réponse à un appel d'offre ou dossier administratif), à partir duquel des travaux de rénovation ou de construction d'usine seront engagés : les chefs de projet de la société de déchets et les chefs de projet de l'entreprise de construction qui interviennent au stade de la conception - les «ingénieurs responsables projet» (IRP) - gèrent des projets de ce type. La réalisation des travaux de rénovation ou de construction d'usine fait également l'objet de projets dont les chefs de projet de la société exploitante et les «ingénieurs responsables d'affaire» (IRA) de l'entreprise de construction sont en charge.

Vingt-deux chefs de projet ont participé à cette enquête : dix sont des chefs de projet de la société exploitante; huit sont des chefs de projet de l'entreprise de construction (quatre en conception et quatre en construction); quatre sont des chefs de projet de la société de déchets. Ces vingt-deux chefs de projet enquêtés sont en majorité des hommes (dixneuf), ayant reçu une formation initiale en science et technique industrielle (seize dont dix ingénieurs diplômés), et dont l'âge varie entre 28 et 56 ans, avec une moyenne de 38 ans (voir encadré 2). La plupart «font du projet» à titre exclusif, quand d'autres - qui se rencontrent dans la société exploitante - en «font» à titre secondaire, en sus de leurs autres responsabilités opérationnelles. Mais tous se reconnaissent dans l'appellation «chef de projet», maîtrisent les méthodes de gestion de projet et en adoptent le vocabulaire.

\section{Encadré 1 \\ La signification des «projets" dans les trois entreprises étudiées}

Les projets consistent ici à construire ou à rénover des usines de production d'eau potable, d'assainissement des eaux usées et de traitement des déchets. L'intervention des entreprises est encadrée par le Code des marchés publics, qui prescrit des rôles formels : maître d'ouvrage; assistant au maître d'ouvrage et maître d'œuvre (ces deux rôles sont dévolus à des bureaux d'étude); entreprise générale de travaux. La société exploitante et la société de déchets sont en position de maître d'ouvrage(1). L'entreprise de construction est une entreprise de travaux (2).

\section{- Dans la société exploitante : gérer l'investissement nécessaire à la réalisation de travaux}

Cette société se compose d'entités territoriales. Son siège, qui réunit les fonctions centrales (directions technique, juridique, financière, des achats, des investissements, etc.), finance et assiste ces entités.

Pour maintenir ses perspectives d'exploitation à long terme, elle finance des travaux de construction de nouvelles usines et de modernisation d'usines existantes. Cette fonction «investissement» est structurée en projets dans la société. Le rôle de leur chef, nommé "Chef de projet d'investissement " (CPI), est de s'assurer que I'utilisation de la dépense par l'entité qui l'emploie est bien conforme aux attentes du siège, qui l'a consentie. Le schéma est le suivant : 1) la collectivité et l'entité s'accordent sur la nécessité d'investir; 2) la direction des investissements autorise la dépense et 3 ) un chef est affecté au projet. II encadre la réalisation des études précédant

(1) La collectivité reste le client final.

(2) Ses clients sont des collectivités, sauf si la collectivité a délégué le service. Dans ce cas, l'entreprise délégataire est son client. Un projet peut donc mettre en scène l'entreprise de construction et la société exploitante (maître d'ouvrage par délégation).

(5) Le groupe emploie environ 80000 salariés, dont $44 \%$ en France, pays où il réalise $36 \%$ de son chiffre d'affaires. En France, les entreprises d'exploitation, de construction et de déchets emploient respectivement 11000,1800 (environ) et 20000 salariés (sources : sites institutionnels de l'entreprise).

(6) Les fiches de poste décrivant le travail de chef de projet dans ces entreprises restent à un niveau de définition très général : ce travail consiste à «piloter» et réclame des compétences d'ordre comportemental («animer», «communiquer», etc.). 
les travaux et s'assure de leur bon déroulement. Il rend compte de son action à la direction des investissements (la procédure d'entreprise l'y oblige). Sur le plan formel, il est subordonné au directeur de l'entité territoriale, lequel affecte un ou deux salariés de l'entité au projet, selon ce qu'il considère être le besoin du chef de projet (il peut s'agir d'un technicien, d'un commercial connaissant bien le client, d'un responsable d'exploitation, etc.). Ces collaborateurs du chef de projet ne lui sont pas subordonnés et le projet n'est jamais leur activité principale. Leur compétence est mobilisée, plus ou moins ponctuellement, sur un aspect circonscrit du projet. Un électricien (technicien) apportera par exemple son éclairage sur les problématiques d'électricité, lors des études précédant les travaux et/ou lors des travaux.

La direction de la société exploitante juge que les investissements sont trop rares pour que les chefs de projet définissent une filière d'emploi autonome ${ }^{(3)}$. En fonction du montant ou de la complexité du projet, le directeur des investissements recrute ou non un chef de projet à temps plein, pour la durée du projet. Si c'est le cas, son recrutement est peu formalisé (il n'est pas toujours recruté dans l'entité qui investit) et sa réaffectation à l'issue du projet est incertaine (puisque son emploi dure le temps du projet) : s'il n'existe pas de nouveau projet sur lequel il pourrait être affecté, il cherche, avec l'appui de la direction des ressources humaines, un autre emploi au sein de la société (ingénieur d'étude, responsable de service, etc.). Quand le projet ne nécessite pas un chef de projet à temps plein, celui-ci est recruté par le directeur de l'entité parmi ses cadres et le projet reste inscrit dans le champ de son activité courante.

On compte vingt-huit chefs de projet en 2011. Tous sont des cadres. Leur ancienneté dans l'entreprise est globalement importante. La moitié est issue de la promotion interne : une partie des métiers de l'exploitation, une autre de la fonction «achats». L'autre moitié est principalement constituée d'ingénieurs ayant exercé dans le domaine des études et/ou de l'exploitation (4).

\section{- Dans la société de déchets : obtenir ou renouveler un arrêté préfectoral d'autorisation}

L'organisation de cette société est similaire à celle de la société exploitante. Elle investit aussi pour construire et rénover des usines. Mais les projets y ont également une autre signification.

Les usines de traitement des déchets s'inscrivent dans un cadre juridique et réglementaire particulier : ce sont des installations classées pour la protection de l'environnement (ICPE). Dans ce domaine, l'engagement de travaux requiert un arrêté préfectoral d'autorisation. L'obtention et le renouvellement de ces arrêtés occupent spécifiquement des chefs de projet, qui sont employés par une entité juridique propre (sorte de filiale-projet de la société de déchets) et sont détachés dans une entité territoriale. Pour faciliter la lecture, ils sont appelés ici "chefs de projet déchets" (CPD). Le directeur de l'entité territoriale désigne parmi ses salariés les membres de l'équipe avec lesquels le chef de projet travaille (cinq ou six personnes). Le chef de projet n'a pas de pouvoir formel sur ses collaborateurs et, s'il se consacre exclusivement au projet, ce projet ne représente qu'une partie de leur activité. Ces collaborateurs sont en général un ingénieur d'étude, un responsable d'exploitation, un responsable "environnement", un responsable de la communication de l'entité, un responsable de travaux (et maintenance). Ils aident le chef de projet à élaborer le dossier d'autorisation, en fonction de leurs compétences. Par exemple, l'activité courante du responsable "environnement » consiste à traiter les questions liées à la réglementation en matière d'environnement, dans l'entité. Cette compétence est utile aux projets, c'est pourquoi il collabore généralement avec les chefs de projet.

On dénombre douze chefs de projet en 2011 dans la filiale-projet de la société de déchets. Tous sont des cadres possédant un diplôme de niveau bac +5 . La plupart sont des ingénieurs, mais certains sont des diplômés d'université ou d'école de commerce; les diplômés d'université n'ont pas tous suivi un cursus technique(5).

\section{- Dans l'entreprise de construction : obtenir un marché de travaux et exécuter les travaux}

L'entreprise conçoit des usines de production d'eau potable ou d'assainissement et les construit si son offre a été retenue à l'issue de la procédure d'appel d'offres. Le chiffre d'affaires associé aux marchés de travaux est au principe de son organisation : ses entités sont des centres de profit distingués en fonction des montants des marchés.

L'entreprise emploie deux types complémentaires de chefs de projet. Le premier, qu'elle appelle «ingénieur responsable projet " (IRP), analyse le cahier des charges spécifiant un appel d'offre et conçoit une offre de travaux. Le second, nommé «ingénieur responsable d'affaire " (IRA), intervient à sa suite : il encadre les sous-traitants qui réalisent les travaux.

Leurs projets mobilisent toujours deux salariés de leur centre de profit qui, comme eux, se consacrent à pleintemps aux projets : un ingénieur d'étude et un commercial (IRP); un ingénieur d'étude et un acheteur (IRA). Les chefs de projet ne les choisissent pas et n'ont pas de pouvoir hiérarchique sur eux. Les équipes sont recomposées projet après projet. Ces chefs de projet ne rendent de compte qu'à leur chef de service, dans leur centre de profit.

Ces projets constituent le cœur d'activité de l'entreprise, qui compte cent cinq chefs de projet (IRP et IRA confondus), en France en $2011^{(6)}$. Ce sont tous des cadres. Ceux qui ne sont pas issus de la promotion interne sont généralement des ingénieurs diplômés.

(3) En 2010-2011, cette direction s'interrogeait toutefois sur l'opportunité d'intégrer la gestion de projet à «l'arbre des métiers » de l'entreprise (Source :

Direction des ressources humaines [DRH], société exploitante).

(4) Source : Direction des investissements, société exploitante.

(5) Source : [Filiale], société de déchets.

(6) Source : DRH, entreprise de construction. 


\section{Encadré 2}

\section{Les propriétés socioprofessionnelles des chefs de projet interrogés et observés}

\section{- Les chefs de projet de la société exploitante}

Cinq des dix enquêtés de la société exploitante sont ingénieurs diplômés. Quatre d'entre eux sont spécialisés en hydraulique et ont débuté à un poste d'ingénieur d'étude - parmi lesquels les deux plus jeunes, qui sont deux trentenaires ingénieurs bac +7 . Le cinquième (45 ans) est docteur-ingénieur (bac +8$)$, spécialisé dans le traitement des eaux usées. Ce dernier, qui sera nommé "Alban", est par ailleurs le seul de ces dix enquêtés qui n'a pas fait l'essentiel de sa carrière dans la société exploitante : il a travaillé quinze ans dans l'entreprise de construction, notamment dans la conception d'usines d'assainissement. Parmi les cinq chefs de projet qui ne sont pas ingénieurs diplômés, deux sont diplômés d'université (bac +5$)$. lls ont moins de 40 ans et leur profil est technique. Spécialisés dans le traitement des eaux usées, ils ont débuté dans le domaine de l'exploitation.

Trois des dix chefs de projet sont issus de la promotion interne. Deux d'entre eux avaient notamment travaillé dans le secteur des achats durant plus de dix ans. C'est le cas du «doyen» des enquêtés (56 ans). En 2008, usé par les déplacements qu'implique son activité d'acheteur, il cherche une nouvelle orientation professionnelle. Ayant appris qu'une entité territoriale dans laquelle il se déplace fréquemment investit et que cet investissement nécessite un chef de projet à temps plein, il sollicite l'affectation au projet auprès du directeur de cette entité et de la direction des investissements, et l'obtient.

Quatre autres enquêtés sont également chefs de projet à temps plein : deux ingénieurs (bac +7 et bac +8 ), un diplômé d'université et un cadre issu du rang - lequel vient des achats comme le «doyen " des enquêtés et est devenu chef de projet dans les mêmes circonstances que ce dernier. Les autres travaillaient déjà dans l'entité quand celle-ci a été autorisée à engager des travaux réclamant un investissement important. Parmi eux, l'un était ingénieur d'étude et un autre travaillait en exploitation. Afin d'élargir le spectre de leurs compétences, ils ont sollicité l'affectation au projet. Le directeur de leur entité a proposé leur candidature au directeur des investissements, qui l'a donc retenue. Quant au troisième - il s'agit du chef de projet ayant été employé par l'entreprise de construction (Alban) -, c'est le directeur de son entité qui a proposé son affectation : le projet présentait une spécificité technique qui lui était familière.

\section{- Les chefs de projet de la société de déchets}

Deux des quatre enquêtés de la société de déchets, ingénieurs diplômés de 28 et de 29 ans, y ont débuté leur carrière - comme ingénieur d'étude - et ont intégré sa filiale-projet en 2009 , après avoir répondu à une offre d'emploi interne. Les deux autres (36 ans chacun) n'ont pas un profil technique. Le premier, diplômé d'école de commerce, a intégré la filiale-projet en 2005 après une première expérience de consultant. Le second, qui sera cité dans ce qui suit sous le pseudonyme de "Victor», détient un master en science politique. Après avoir débuté en 1997 comme attaché parlementaire - il a notamment travaillé avec les rapporteurs du budget du ministère de l'Environnement -, Victor intègre en 2002 un cabinet de conseil en stratégie et concertation spécialisé dans l'aménagement du territoire et l'environnement. En 2008, au terme d'une mission menée pour la société de déchets, un poste dans la filiale-projet lui est proposé, qu'il accepte.

\section{- Les chefs de projet de l'entreprise de construction}

Parmi les quatre chefs de projet de l'entreprise de construction qui interviennent au stade de la conception de travaux (IRP), un seul a plus de 30 ans (il est âgé de 46 ans) et a connu d'autres employeurs. Après avoir travaillé dans une entreprise de travaux publics, il entre dans cette entreprise en 1999, comme dessinateur. II devient technicien de bureau d'études en 2003, puis chef de projet en 2009. Deux des trois autres sont des ingénieurs diplômés de la même école - spécialisée dans l'environnement. L'un d'entre eux est le seul de tous les enquêtés à avoir débuté sa carrière au poste qu'il occupait lors de l'enquête. Le dernier chef de projet possède une licence et a débuté dans l'entreprise comme technicien dernier échelon.

Les quatre chefs de projet de l'entreprise de construction qui encadrent la réalisation de travaux (IRA) sont plus âgés : deux ont 35 ans, un autre a 40 ans et un dernier a 47 ans. Ce dernier («René») est un ingénieur diplômé (47 ans) d'une école généralement tenue pour "prestigieuse". C'est en 2003, après une carrière de fonctionnaire, qu'il entre dans l'entreprise comme chef de projet. Les marchés de travaux dont il est chargé engagent des montants qui sont bien plus élevés que ceux dont ses homologues sont responsables. Un autre de ces chefs de projet est une femme : Marie, 35 ans, qui est titulaire d'un master en sciences et techniques de l'environnement, a travaillé cinq ans dans une PME (petite et moyenne entreprise) avant d'intégrer le centre de recherche de l'entreprise de construction (comme ingénieur de recherche), en 2006. Fin 2008, Elle répond à une offre d'emploi interne et devient chef de projet. Les deux autres chefs de projet (35 et 40 ans) ne sont pas diplômés du supérieur. Ils sont entrés dans l'entreprise comme dessinateurs, après avoir occupé divers emplois dans de petites sociétés industrielles. 
Le tableau ci-après synthétise les propriétés socioprofessionnelles des chefs de projet qui ont participé à cette enquête :

\begin{tabular}{|c|c|c|c|c|c|c|}
\hline $\begin{array}{l}\text { Chefs de projet } \\
\text { Société }\end{array}$ & $\begin{array}{c}\text { Sexe } \\
\text { masculin } \\
\text { (féminin) }\end{array}$ & $\begin{array}{l}\text { Âge moyen } \\
\text { (plus jeune/ } \\
\text { plus âgé) }\end{array}$ & $\begin{array}{c}\text { Diplôme } \\
\text { niveau bac }+5 \\
\text { (plus) }\end{array}$ & $\begin{array}{l}\text { Formation initiale en } \\
\text { science et technique indus- } \\
\text { trielle (dont ingénieurs) }\end{array}$ & $\begin{array}{l}\text { Accès au poste par } \\
\text { promotion interne } \\
\text { dans l'entreprise }\end{array}$ & Total \\
\hline Société exploitante & $8(2)$ & $<43$ ans $(29 / 56)$ & $7(3)$ & $7(5)$ & 3 & 10 \\
\hline $\begin{array}{l}\text { Entreprise de } \\
\text { construction }\end{array}$ & $7(1)$ & $<37$ ans $(28 / 47)$ & $4(0)$ & 7 (3) & 4 & 8 \\
\hline Société de déchets & $4(0)$ & $>32$ ans $(28 / 36)$ & $4(0)$ & $2(2)$ & - & 4 \\
\hline Total & $19(3)$ & $>38$ ans $(28 / 56)$ & $15(3)$ & $16(10)$ & 7 & 22 \\
\hline
\end{tabular}

L'observation de ces chefs de projet en action, l'examen du sens qu'ils donnent à ce qu'ils font et le recoupement des descriptions qu'ils livrent de leur activité (7) révèlent des pratiques et des savoirfaire communs à tous, irréductibles à l'objet de leur projet ou à l'identité de leur employeur. La spécificité de la «fonction projet» est d'ailleurs reconnue par deux des filiales, tandis que cette reconnaissance est en discussion dans la troisième ${ }^{(8)}$. Ces pratiques et ces savoir-faire communs, qui seront décrits et analysés tout au long de l'article, constituent la «substance» du groupe professionnel des chefs de projet ${ }^{(9)}$. L'article s'inscrit dans la perspective théorique proposée par Florent СнамРY (2009, 2011) : il fait porter la focale sur l'unité du groupe des chefs de projet, du point de vue du contenu de leur travail.

Ces contenus de travail seront plus précisément analysés à partir des caractéristiques propres à ce que CHAmpY nomme les «professions à pratique prudentielle», en référence à l'acception aristotélicienne de la prudence : un «art de conjecturer sur le contingent et sur le possible dans une situation donnée», qui «s'exerce sur le terrain de l'action» (Granjon, 1999, p. 138). D'après Champy, le professionnel prudentiel traite de cas dont le caractère «singulier» et «complexe» rend «incertain» le résultat de son travail (CHAmpy, 2009, 2011). De surcroît, les «exigences morales et cognitives » de son activité, qui sont les critères d'évaluation de son travail, peuvent entrer en tension et, dans certains

(7) On retrouvera une présentation complète du protocole d'enquête dans l'annexe, à la fin de l'article.

(8) Source: «Projet Gestion des chefs de projet», DRH Groupe (2008). Classiquement, le groupe identifiait deux grandes filières d'emploi : l'expertise technique et le management général (chef de service, directeur d'entité, etc.). Une réflexion sur la filière «gestion de projet» a émergé au milieu des années 2000, traduisant une reconnaissance progressive de la spécificité de cette fonction. La société exploitante est la seule entreprise étudiée dans laquelle cette reconnaissance ne se concrétise pas dans l'existence d'une filière spécifique, mais ce point fait débat dans l'entreprise depuis 2010 (voir encadré 1). (9) Une conception substantialiste des groupes professionnels «met l'accent sur l'unité des groupes étudiés » (CHAmpy, 2009, p. 95). СнамPY invite à «saisir les caractéristiques générales de l'activité» et à les rapporter, dans un second temps, aux éléments de diversité interne du groupe considéré. Selon lui, c'est ainsi que l'on peut «penser ensemble» l'unité et la diversité des groupes professionnels (pp. 141-142). cas, il doit arbitrer entre ces exigences sans disposer d'appui pour fonder son choix. Son travail recèle pour ces raisons une dimension «conjecturelle» (Ibid.). Dépourvu de certitude quant aux conséquences de ses choix, ce professionnel doit en effet appréhender la globalité du processus dans lequel il est impliqué, conduire des délibérations et se référer à son expérience, avant de prendre la décision qu'il aura jugée équilibrée (compte tenu des circonstances). Cette décision suppose qu'une «autonomie de réflexion» (Ibid.) lui ait été ménagée. C'est pourquoi les métiers prudentiels sont en général des professions à statut: le statut protège l'autonomie dans le travail, mais aussi l'autonomie du groupe pour définir la finalité de l'activité et la manière de l'exercer.

En France, la gestion de projet n'est pas un métier protégé; son exercice n'est pas réglementé. Les chefs de projet étudiés sont affectés à leurs projets par une hiérarchie, qui, globalement, ne leur impose pas de modes opératoires, mais qui contrôle et valide un travail dont les principaux jalons sont fixés par une procédure ${ }^{(10)}$. Ils exécutent des décisions peu ou prou stratégiques sans avoir participé à leur élaboration, comme la plupart des cadres (KarVar, rouban, 2004). Pour autant, l'idéal-type de la «pratique prudentielle» se révèle particulièrement heuristique pour rendre compte de ce groupe professionnel. Ce faisant, nous rejoignons une piste suggérée par СНАмPy lui-même : «[...] certains métiers, à l'instar des policiers, des militaires ou des travailleurs sociaux, ont des contenus du travail qui se prêteraient à une pratique prudentielle. Les comparer à l'idéal type de la pratique prudentielle peut ainsi être particulièrement éclairant, notamment pour saisir les effets sur leur travail des tensions entre les valeurs servies et les objectifs poursuivis dans l'activité» (2009, pp. 188-189).

(10) Si la procédure d'entreprise qui encadre la gestion des projets est spécifique à chaque entreprise, elle traduit dans toutes une «exigence de forme» (СосHOY, 2002) : elle définit la succession des opérations qui scandent la réalisation du projet, mais n'impose pas (ou très peu) de modes opératoires. La procédure organise surtout le contrôle de ce travail : la gestion des projets de tous les chefs de projet étudiés est jalonnée de réunions imposées avec leur hiérarchie. 
Le cas des chefs de projet prouve précisément que la pratique d'un métier non protégé peut s'analyser à partir des catégories de l'activité prudentielle. L'étude du contenu du travail de ces chefs de projet révèle en effet qu'ils adoptent un regard global sur leur projet, surveillent les contingences pouvant entraver son déroulement, mobilisent un «sens du possible» pour résoudre les problèmes singuliers et complexes auxquels ils se heurtent et pour arbitrer entre des exigences contradictoires. Si le lien de subordination inhérent au statut salarié contraint leur autonomie, on verra aussi qu'il leur procure un certain nombre d'appuis organisationnels confortant la construction de leur vue d'ensemble, de leur vigilance et de leur sens du possible.

\section{Construire une vue d'ensemble pour "porter" le projet}

Les entretiens et les observations montrent qu'il est tout d'abord attendu du chef de projet qu'il soit capable de «porter» le projet face à des audiences aux exigences variées: collaborateurs internes, hiérarchie, entreprises sous-traitantes, services de l'État, élus du territoire. Il doit à cet effet s'appuyer sur une vue d'ensemble de son projet.

Celle-ci articule trois dimensions : une dimension stratégique, qui renvoie au but du projet; une dimension technique, qui se matérialise dans la construction d'un plan de coordination des tâches dont la réalisation est nécessaire à l'atteinte du but; une dimension sociale, qui consiste à envisager le projet comme une séquence d'un ensemble qui le dépasse.

\section{La dimension stratégique : les propriétés essentielles du projet}

Par définition, le travail d'un chef de projet est orienté vers une «cible», c'est-à-dire vers un but précis, visé par son employeur (GAREL, 2011). Les chefs de projet étudiés sont formellement responsables de l'atteinte de la "cible» définissant leur projet. Les uns doivent ainsi obtenir un arrêté préfectoral (pour une usine de déchets) tandis que les autres sont chargés de remporter un marché de travaux ou de livrer une usine (neuve ou rénovée) à une collectivité.

Toute cible se décline en un «triptyque d'objectifs » : délai, qualité, coût (GAREL, 2011). Les chefs de projet étudiés n'échappent pas à cette règle. Pour l'essentiel, le contenu des objectifs que leur fixe leur employeur s'impose à eux - l'équilibre entre le triptyque de contraintes est en effet défini en amont de leur affectation au projet. Le respect des objectifs de délai, de qualité et de coût est le premier critère de l'évaluation de leur travail par leur hiérarchie. Détaillons la manière dont ces objectifs se présentent à eux.
Les chefs de projet des trois entreprises étudiées sont tous tenus de respecter une échéance. Le projet auquel ils sont affectés doit être achevé à une date précise, qu'ils n'ont pas la possibilité de modifier. Cette date peut être, par exemple, la date fixée par la procédure de marché public pour la remise d'une offre ou bien celle convenue contractuellement avec la collectivité pour la mise en service de l'usine. Le non-respect de cette exigence entraîne l'éviction du marché, ou d'éventuelles pénalités pour chaque jour de retard.

Les projets dont il est question ici sont toujours associés, parfois en amont, à une réalisation technique: une usine est construite ou une usine existante est modernisée. Cette réalisation doit satisfaire un besoin de la collectivité considérée (améliorer la qualité de l'eau potable ou absorber un surplus de déchets produits sur le territoire, par exemple). Elle implique en général un certain nombre d'exigences techniques, fixées par la réglementation ou le client. Pour une usine de production d'eau potable par exemple, elles renverront à un volume de production et à un niveau de qualité de l'eau. Elles concerneront ensuite des garanties en matière d'efficacité : caractère fonctionnel de l'usine, fiabilité des équipements, du process, des matériaux, etc. Les données techniques des projets s'imposent là encore aux chefs de projet des trois entreprises ${ }^{(11)}$.

Tous ces chefs de projet doivent intégrer une contrainte financière. Par exemple, le directeur des investissements de la société exploitante définit le montant de l'investissement qu'il juge nécessaire pour réaliser des travaux; ce montant constitue le budget dont le chef de projet qui a été affecté à la gestion de l'investissement en question dispose pour rétribuer les entreprises qui les réalisent. Quant aux chefs de projet de l'entreprise de construction qui conçoivent des offres de travaux - les «ingénieurs responsable projet» $(\text { IRP })^{(12)}$-, ils raisonnent à partir d'un budget estimé, qui figure dans l'appel d'offre auquel ils répondent. Leur proposition devant préserver la rentabilité du projet pour leur employeur, une partie de leur activité consiste à minimiser le coût des travaux tout en respectant les exigences en matière de performance.

Cette dimension de la vue d'ensemble renvoie à un premier rôle formel des chefs de projet, qui consiste à «garder le cap» sur la cible et ses

(11) En pratique, il peut arriver que les entreprises des chefs de projet étudiés convainquent leur client de modifier les données techniques d'un projet. Ce point mériterait à lui seul d'importants développements. D'une certaine manière, il pourrait appuyer une critique de la distinction entre «autonomie de décision» et "autonomie de réflexion», distinction à laquelle ChAмpY procède (2011). Nous n'en dirons rien ici, parce que le travail qui consisterait à convaincre un client de revoir l'expression de son besoin ne relève pas de la responsabilité des chefs de projet étudiés.

(12) Voir encadré 1 (en introduction de l'article). 
contraintes. C'est pourquoi, explique l'un d'entre eux ${ }^{(13)}$, ils «repasse[nt] ces points en revue à chaque problème» qu'ils rencontrent. Mais ces exigences sont liées - un retard engendre un surcoût, par exemple - et l'équilibre est menacé par une foule d'événements, si bien qu'il leur faut parfois se résoudre à privilégier l'une de ces exigences au détriment d'une autre. Nous y reviendrons.

\section{Le plan de coordination : la dimension technique de la vue d'ensemble}

Chaque chef de projet étudié, quelle que soit par ailleurs la cible de son projet, établit un plan de coordination, à partir des exigences techniques, financières et temporelles du projet auquel il a été affecté. Dans les trois entreprises, la construction de ce plan est une des toutes premières étapes par laquelle la procédure impose de passer. À ce stade, les chefs de projet envisagent la division du travail de réalisation de leur projet, entendue dans sa dimension technique. L'enjeu est ici de définir l'ordre des interventions des différents acteurs du projet, appelés à contribuer à sa réalisation à hauteur de leur compétence. Il s'agit des autres salariés de l'entreprise du chef de projet considéré et des salariés de ses partenaires commerciaux. Les projets étudiés, en effet, «ignore[nt] les frontières des métiers, mais aussi des entreprises» (SEgrestin, 2004). Dans les trois entreprises investiguées, les chefs de projet ne peuvent agir qu'à la marge sur le nombre de ces acteurs. Le choix du nombre des entreprises qui concourent à leur projet, quand il n'est pas contraint par le cadre juridique et réglementaire dans lequel il s'inscrit ${ }^{(14)}$ et n'a pas été arrêté avant leur affectation au projet, est en dernière analyse du ressort de leur hiérarchie; le choix du nombre de collaborateurs internes (salariés de la même entreprise que ces chefs de projet $\left.{ }^{(15)}\right)$ l'est également.

Dans ces conditions, les chefs de projet de ces trois entreprises commencent par recenser toutes les tâches nécessaires à la réalisation du projet auquel ils ont été affectés (à partir des données qui le décrivent) et par se figurer les contraintes (technique, financière, temporelle) propres à chacune de ces tâches. Ils évaluent ensuite leur degré d'interdépendance et pensent la logique de leur articulation. Pour mener à bien ce travail d'évaluation et de réflexion, ils s'appuient sur les dispositifs informatiques de gestion de projet (Microsoft Project,

(13) Entretien du 12 juillet 2010 (chef de projet d'investissement, société exploitante).

(14) Le Code des marchés publics oblige par exemple la société exploitante à "passer » un marché de travaux en «lots séparés », c'est-à-dire à le partager entre plusieurs entreprises de travaux, si ce marché implique des «prestations distinctes» (article 10). (15) Voir encadré 1. par exemple) (16). Une représentation schématique de l'avancement du projet en résulte, qui combine l'articulation des tâches avec leur durée respective. L'extrait d'entretien avec Marie, qui occupe un poste d'«ingénieur responsable d'affaire» (IRA) dans l'entreprise de construction (17), montre que l'utilisation d'un outil de représentation permet de détecter a priori les tâches - dites «critiques» - qui devront faire l'objet d'une attention toute particulière quand la réalisation du projet sera effectivement engagée :

"On aura telle tâche de l'ingénieur [de l'équipeprojet] qui dure trois mois. On fait un rétro planning à partir de là, pour voir les tâches critiques : l'achat de tel équipement par exemple, ne peut plus être passé après telle date; ça signifie qu'il faut lancer les appels d'offre avant telle date, donc que l'ingénieur ait fait les spécifications, et ainsi de suite.... Je construis le planning d'affaire, qui sert à visualiser les tâches qui vont s'enchaîner jusqu'à la réception de l'installation. »

(Marie, 35 ans, IRA, entreprise de construction $\left.{ }^{(18)}\right)$.

On le voit, Marie coordonne a priori les interventions de tous les contributeurs au projet, en partant de l'échéance fixée pour ce dernier ( $\ll$ on fait un rétro planning »). C'est ainsi qu'elle peut identifier les «tâches critiques», c'est-à-dire celles dont le retard dans l'exécution engendrerait inévitablement un dépassement du délai du projet.

De manière générale, la construction du plan de coordination est une étape du travail des chefs de projet étudiés qui est imposée par la procédure de leur entreprise. Celle-ci prévoit également que le plan ainsi construit fasse l'objet d'une validation par leur hiérarchie, validation qui enclenche la mise en route effective du projet. Les chefs de projet vérifient alors quotidiennement son application, veillant à ce que les tâches des contributeurs (internes et externes à leur société), comme les pièces d'un puzzle, s'emboîtent conformément au plan.

En pratique, l'une des difficultés de leur travail réside dans le fait que ce versant technique de la vue d'ensemble les oblige à se représenter l'articulation des diverses contributions dans ses moindres détails. Le savoir-faire d'un chef de projet se révèle alors dans sa capacité à opérer les allers-retours indispensables entre, d'une part, la vision globale que traduisent le plan de coordination et les caractéristiques essentielles du projet, et, d'autre part, la multitude de détails qui risquent à tout moment d'en bloquer la réalisation. Écoutons ce qu'en dit René,

(16) La méthode PERT (Project Evaluation and Review Technique) et le diagramme de Gantt, qui sont des méthodes complémentaires de planification utilisées dans tous les projets, sont intégrées aux outils informatiques de la gestion de projet. (17) Marie est donc chargée d'encadrer l'exécution de travaux de rénovation ou de construction d'usines (voir encadré 2).

(18) Entretien du 18 juin 2010. 
chef de projet de l'entreprise de construction qui, comme Marie, encadre la réalisation de travaux :

"Je ne suis expert en aucun domaine, j'ai la vision globale du projet [construction]. Je ne peux donc pas laisser un expert [technique] s'égarer: "Je vais vous mettre un truc super”. Je dirais : "Attendez ... derrière, j'ai une petite fenêtre! Il faut que ça s'intègre”. »

(René, 47 ans, IRA, entreprise de construction $\left.{ }^{(19)}\right)$.

Certes, René se figure bien l'articulation des contributions à la réalisation de son projet, conformément à ce que sa hiérarchie attend de lui. Mais cette «vision globale» n'est opératoire que si, dans le même temps, il mobilise une «connaissance experte» (Merchiers, Pharo, 1990) ${ }^{(20)}$ de son projet : il doit être capable de «saisir dans la situation les détails importants » (Ibid., p. 103) dont fourmille son projet ( "j'ai une petite fenêtre») et de les resituer dans la perspective d'ensemble ( $«$ il faut que ça s'intègre»). C'est à cette condition qu'il peut faire respecter le plan de coordination.

Si tous les chefs de projet étudiés doivent pouvoir identifier «dans la situation» les détails susceptibles de se répercuter négativement sur le déroulement de leur projet, ils doivent aussi et surtout s'efforcer de le faire avant que la réalisation effective du projet n'ait débuté. Le cas d'Alban, chef de projet d'investissement (société exploitante), est significatif à cet égard. Son projet, qui devait déboucher sur la construction d'une usine d'assainissement des eaux usées, était déjà bien avancé lorsqu'il a dû partager son expérience avec ses pairs lors des «Journées chefs de projet d'investissement» de $2010^{(21)}$. Il $\mathrm{y}$ exposa un problème auquel il se heurtait, après avoir précisé qu'il voulait insister sur l'importance du détail dans la conduite de son travail :

«Depuis quelques semaines, j'ai des problèmes à cause... des parasols qu'on doit mettre sur le toit de la station! En fait, ça entraîne une succession de problèmes qu'on n'a pas anticipés. Au début, ce n'est pas à ça qu'on pense! On ne réalise pas qu'une chose a priori insignifiante peut avoir d'importantes répercussions.»

(Alban, 45 ans, CPI, société exploitante [s'adressant à ses pairs]).

Le projet d'Alban engage un montant d'investissement élevé; le procédé technique utilisé est innovant. Or, si la pose de parasols est «insignifiante» au regard de ces considérations, il reste

(19) Entretien du 6 septembre 2010.

(20) La «connaissance experte» est définie par ces auteurs comme une «capacité à saisir dans la situation les détails importants pour l'activité» (p.103).

(21) La direction des investissements organise des «Journées» annuelles, sur deux jours, au cours desquelles les chefs de projet échangent leurs expériences et écoutent les interventions de cadres du siège de leur société. Les «Journées» d'octobre 2010 et d'octobre 2011 furent observées, de même qu'une formation de trois jours à la gestion de projet d'investissement en juin 2010. qu'elle a une influence sur d'autres tâches, freinant - entre autres «problèmes» - la finition de travaux attenants. Alban juge donc qu'il aurait fallu en tenir compte dès le «début» du projet. Le problème soulevé ici a déjà été identifié. Plus le projet avance et plus la connaissance qu'en a le chef de projet s'affine, mais plus, aussi, sa capacité d'action (sur le projet) se réduit, en raison de l'irréversibilité croissante des décisions prises; "au début», au contraire, sa capacité d'action est grande, mais sa connaissance du projet, elle, est faible (MidLer, 1993). Christophe MidLer insiste sur l'importance, dans ces conditions, de la «collecte d'informations » en amont de la réalisation du projet. Insistons ici sur cet enjeu crucial du travail de chef de projet : identifier a priori les «détails importants » du projet, ceux qui nécessitent une vigilance bien particulière du fait qu'ils peuvent affecter son déroulement - voire empêcher l'atteinte de la cible ${ }^{(22)}$.

\section{Envisager le projet comme une séquence du travail global}

La troisième dimension de la vue d'ensemble renvoie à la trajectoire des projets. Ces projets débouchent, on l'a dit, sur la création ou la modernisation d'une usine. Tous les chefs de projet étudiés sont dépositaires de ce que l'on peut appeler, en s'inspirant des travaux de Philippe LefEBvRE (2003) sur la production dans les manufactures de la fin du XVIIIe siècle, «les contraintes du travail global»(23). Prenons le cas de la construction d'une nouvelle usine de production d'eau potable: un chef de projet conçoit l'usine, un second finance sa construction, un troisième la construit, et enfin, un exploitant, l'utilisateur final de l'usine, la valorise pour le compte de la collectivité. Le «travail global» désigne ici la séquence conception-constructionexploitation. Tous les chefs de projet étudiés sont explicitement tenus d'envisager la conduite de leur travail à l'aune des contraintes des opérations situées en aval. Quand la procédure d'entreprise ne le prescrit pas, leur hiérarchie s'assure que les contraintes des acteurs placés en aval de leur position dans la séquence du travail global sont bien prises en considération.

Au sein de l'entreprise de construction, l'injonction à l'intégration des contraintes de l'aval est inscrite dans la procédure à suivre en matière de gestion des projets. Par exemple, un chef de projet chargé de l'exécution de travaux (IRA) doit successivement «préparer», «démarrer», «réaliser» et

(22) Ce point fera l'objet de plus amples développements dans la seconde partie, dédiée à la vigilance.

(23) L'auteur explique que ces manufactures rassemblaient des métiers - au sens fort de l'Ancien Régime - sans les transformer ni grever leur indépendance pour définir leurs modes opératoires. Pour que leurs interventions successives puissent aboutir au produit final, il fallait que chaque intervenant intègre, dans la conduite de son travail, les contraintes des intervenants placés en aval. 
«terminer» son «affaire» ${ }^{(24)}$. Quand ce chef de projet «prépare» son «affaire», il doit (notamment) «intégrer l'exploitant» (25). Dans la société de déchets, le directeur de l'entité territoriale désigne les salariés de l'entité qui devront appuyer le chef de projet chargé d'obtenir l'arrêté préfectoral. Un ingénieur du service travaux et un responsable d'exploitation figurent généralement parmi ces salariés : les «acteurs aval» sont bien intégrés de façon «précoce» (et formelle) au projet - c'est là une recommandation classique en gestion de projet (GAREL, 2011). Dans la société exploitante, nulle procédure formelle ni pratique d'usage : les chefs de projet, globalement plus âgés que les autres et aux parcours professionnels variés, puisent dans leur expérience. Lors des entretiens, certains affirmaient travailler en étroite relation avec un responsable d'exploitation sans que leur supérieur hiérarchique ait eu à le leur suggérer; pour d'autres, le directeur de l'entité avait affecté un responsable d'exploitation à leur projet; d'autres enfin, qui pouvaient mobiliser une expérience personnelle, n'associaient de fait aucun responsable d'exploitation à leur travail (26). Quoi qu'il en soit, la hiérarchie de tous ces chefs de projet doit souvent rappeler la nécessité de cette intégration. Illustrons-le en nous appuyant sur une partie du contenu d'une réunion de chantier observée le 29 juillet 2010.

Le projet ayant fait l'objet de cette réunion de chantier consistait à réhabiliter une vieille usine d'assainissement des eaux usées. Le supérieur hiérarchique du chef de projet chargé de réaliser ces travaux de réhabilitation ${ }^{(27)}$ s'était déplacé pour la réunion. Au cours de la visite du chantier l'ayant précédée, les trois participants - le chef de projet (IRA), son supérieur hiérarchique et le représentant de l'exploitant de l'usine - s'arrêtèrent sur un équipement tout juste achevé, qui ressemblait à une tranchée. Ils en considérèrent d'abord le fond un instant, puis le supérieur du chef de projet s'empara d'un bout de bois jonchant le sol, à l'aide duquel il tenta - en vain - de toucher ce fond. Ayant constaté de cette manière que l'équipement n'était pas fonctionnel - car «trop étroit» et «trop profond»-, il s'emporta contre le concepteur du projet, avant de reprocher à son subordonné d'avoir insuffisamment

(24) Source : Procédure, «Projets de Construction», 2010.

(25) La procédure n'impose aucune modalité d'intégration. La définition de ces modalités est laissée à l'appréciation des chefs de projet et de leur hiérarchie.

(26) Ceux-là avaient précisément travaillé dans le domaine de l'exploitation avant de devenir chef de projet. Ils engageaient donc leur compétence en matière d'exploitation dans la conduite de leur travail de chef de projet. Tous les chefs de projet interrogés et observés ont tendance à investir leur spécialité première dans leur travail. Nous reviendrons sur ce point dans la dernière partie du texte.

(27) Ce chef de projet est donc un «ingénieur responsable d'affaire» (IRA) de l'entreprise de construction. resitué son travail dans la séquence globale du travail :

«L'exploitant, ensuite, il doit nettoyer [l'intérieur de l'équipement]!»

Selon le supérieur du chef de projet, l'état de l'équipement était donc de nature à compliquer le travail de l'exploitant de l'usine réhabilitée. Il en profita pour se justifier auprès du représentant de l'exploitant :

«Je me bats sur toutes mes affaires... je dis à mes IRA [chefs de projet "construction"] de bien tenir compte des problèmes de l'exploitant.»

L'adoption d'une vue globale sur le projet, entendue dans ses trois dimensions, conditionne la capacité des chefs de projet à représenter le projet, notamment vis-à-vis de la collectivité locale concernée et des services de l'État. Dans l'exercice quotidien de leur travail, elle permet de réduire l'incertitude sur l'issue du projet, c'est-à-dire d'anticiper les problèmes susceptibles d'entraver son avancement. Ce point fait l'objet de la partie suivante.

\section{Anticiper les problèmes pour contenir l'incertitude}

Les projets qui occupent les chefs de projet étudiés sont des objets complexes. En premier lieu, ils intègrent des variables techniques, financières, contractuelles, administratives, juridiques et réglementaires qui sont en partie liées entre elles. En second lieu, rappelons-le, les exigences associées à leur cible sont imbriquées. Un retard signifie généralement un coût supplémentaire; la qualité technique coûte cher. En troisième lieu, les multiples tâches concourant à la réalisation des projets sont articulées les unes aux autres et une foule de détails sont susceptibles de nuire à la logique de leur articulation. Enfin, les décisions prises sont de plus en plus irréversibles à mesure que cette réalisation avance.

Ces projets présentent également, au-delà des similitudes observables d'un projet à l'autre, une part irréductible de singularité. Les individus qu'ils mobilisent ont en effet des personnalités et des trajectoires variées, et leurs comportements sont parfois imprévisibles. De plus, ces projets s'insèrent dans des tissus territoriaux aux propriétés pour partie singulières, et les terrains sur lesquels les travaux sont effectués sont toujours particuliers.

Pour ces raisons - singularité et complexité des projets -, les chefs de projet étudiés s'attachent à borner le caractère incertain de l'issue de leur projet. Ils le font principalement en articulant deux formes complémentaires de vigilance. 


\section{Choisir a priori les aspects du projet sur lesquels concentrer sa vigilance}

La première stratégie consiste à exercer une fonction de vigilance orientée sur certains aspects ou sur certains détails du projet. En orientant ainsi leur vigilance, les chefs de projet délaissent nécessairement d'autres aspects ou détails de leur projet. Leur vigilance devrait en principe être constante dans le temps et exhaustive, mais, en admettant que ce soit possible ${ }^{(28)}$, ils ne disposent pas du temps nécessaire à cette constance et à cette exhaustivité. Ils arrêtent donc a priori les aspects et les détails sur lesquels déployer spécialement leur vigilance, soit qu'ils jugent qu'ils ont une plus grande influence que d'autres sur le déroulement de leur projet, soit qu'ils pensent qu'un problème éventuel qui leur serait lié serait particulièrement préjudiciable à l'atteinte de leur cible. Par exemple, pour Victor et ses collègues chefs de projet de la société de déchets, ce sont les délais réglementaires qui priment :

"La publication d'un avis d'enquête publique dans la presse, c'est quinze jours avant le début de l'enquête, pas quatorze; si c'est quatorze, on annule, on refait. C'est arrivé. Un journal a publié l'avis quatorze jours avant : ils ont pensé que, lundi ou mardi, c'était pareil! Il faut être particulièrement attentif à la forme. »

(Victor, 36 ans, CPD, société de déchets ${ }^{(29)}$ ).

L'objet de cette vigilance particulière diffère selon les cibles des projets. Mais, quelle que soit leur cible, tous les chefs de projet étudiés «enferme[nt] [leur] perception sur un certain nombre de phénomènes pouvant indiquer une menace» (BRION, 2005, p. 107); tous concentrent leur vigilance sur un ou plusieurs aspects de leur projet, au détriment de certains autres. L'extrait cité ci-dessus montre que, pour choisir les phénomènes sur lesquels «enfermer leur perception», les chefs de projet s'appuient non seulement sur leur expérience, mais aussi sur celle de leurs pairs («c'est arrivé»), dont l'entreprise assure la capitalisation et la dissémination. Contribuer au partage des expériences et partant, au ciblage de la vigilance, constitue une tâche à part entière de leur travail.

Dans la société de déchets, la procédure prescrit la tenue de réunions formelles, mensuelles, au cours desquelles les chefs de projet échangent leurs expériences, sous l'égide de leur supérieur hiérarchique (le directeur de la filiale projet de cette société). La procédure dans l'entreprise de construction prescrit la même chose aux chefs de projet intervenant au stade de la conception (IRP). Quant aux chefs de projet qui réalisent les travaux (IRA), ils rendent compte de leur action au chef de leur service au cours d'entretiens formels dont la tenue régulière est prévue par la procédure. Ce chef de service, qui

(28) La vigilance peut être considérée comme étant «intermittente par nature» (BRION, 2005).

(29) Entretien du 16 novembre 2010. a une vision d'ensemble de la totalité des projets gérés dans son centre de profit, peut ainsi attirer leur attention sur certains aspects de leurs projets respectifs. Enfin, la procédure dans la société exploitante oblige les chefs de projet à rédiger des rapports trimestriels destinés à la direction des investissements située au siège, dans lesquels ils recensent les problèmes rencontrés. Si cette direction est parvenue à identifier la cause d'un problème récurrent, elle émet une recommandation, venant orienter leurs choix relatifs aux aspects de leur projet sur lesquels concentrer leur vigilance. C'est de cette manière qu'ils ont su, par exemple, qu'ils devaient être particulièrement attentifs à la qualité des études précédant l'engagement des travaux, mais aussi que certaines études étaient plus importantes que d'autres ${ }^{(30)}$.

Dans ces entreprises, l'organisation de la capitalisation des savoirs produits dans la gestion des projets permet à chaque chef de projet d'orienter sa vigilance. Elle est aussi un appui crucial pour résoudre les problèmes qui apparaissent à mesure qu'avance la réalisation du projet. Nous y reviendrons dans la dernière partie du texte.

\section{Tenter de préserver des marges de manœuvre pour faire face à l'imprévu}

En dépit du cadrage initial et des efforts de vigilance, des problèmes de tous ordres surgissent inévitablement. Cet état de fait requiert un type spécifique de vigilance, consistant à «se tenir prêt à réagir vis-à-vis d'un événement dont on ne sait quand il se produira, ni sous quelle forme il surviendra ou même s'il arrivera» (Duval, 1990, p. 123). Un chef de projet qui exerce cette vigilance sait que des problèmes apparaîtront - sans qu'il sache a priori lesquels - et qu'un problème, quelles qu'en soient par ailleurs la nature et l'origine, est majeur s'il implique un coût supplémentaire, un retard important ou encore, s'il peut conduire à gêner le travail des acteurs placés en aval. C'est donc parce qu'il le sait qu'il tente de conquérir un surcroît de marge de manœuvre, de dégager des ressources supplémentaires pour remédier aux probables difficultés futures. De fait, ces ressources sont bien souvent financières. C'est notamment le cas pour Alban, chef de projet d'investissement (société exploitante), qui explique à propos de son projet :

«Je m'arrange pour me créer un montant que je pourrai utiliser librement. Je le fais en transparence avec la direction des investissements. En fait, sur ce projet, [X \%] des travaux ne sont pas encore alloués (c'est significatif). Je ne peux pas changer les budgets

(30) Lors des «Journées chefs de projet d'investissement» et lors de la formation observée en 2010, l'adjoint du directeur des investissements insista sur la nécessité d'exercer une vigilance toute particulière sur la qualité d'une étude spécifique, celle qui consiste à évaluer la qualité du sol sur lequel les travaux doivent être réalisés. 
alloués aux marchés signés, mais tous les travaux ne sont pas encore alloués : j'ai parié que je les ferai pour moins et gardé la différence. »

(Alban, 45 ans, CPI, société exploitante ${ }^{(31)}$ ).

Quand un chef de projet de la société exploitante est nommé pour gérer un investissement, les entreprises de travaux peuvent être déjà toutes choisies : ce chef de projet n'a pas de marge de manœuvre. Mais, dans le cas d'Alban, une part «significative» de marchés de travaux ne sont pas alloués; il a alors pu se ménager une marge de manœuvre financière en «pariant» que ces marchés seront alloués à un prix plus faible que prévu ${ }^{(32)}$. Ce surcroît de latitude financière est un matelas supplémentaire pour faire face à l'imprévu.

Les chefs de projet étudiés n'ont pas toujours la possibilité de dégager de telles marges de manœuvre. Mais, lorsqu'ils l'ont, ils ont tendance à s'en saisir. Notons aussi le caractère conjecturel du choix d'Alban : il perdrait son pari si les travaux étaient finalement attribués au prix prévu.

\section{Mobiliser son "sens des réalités" pour résoudre les problèmes}

Les problèmes qui peuvent se poser aux chefs de projet dans la gestion effective de leur projet sont multiples. Un problème peut être prévisible ou imprévisible. Il peut se rencontrer dans la plupart des projets comme être spécifique à un projet particulier et se révéler inédit. Il peut être complexe - au point que ses données soient irréductibles à une description exhaustive -, du fait de l'inextricable enchevêtrement des variables à intégrer. Il peut enfin influer sur le plan de coordination jusqu'à mettre en péril l'atteinte de la cible et contraindre à sacrifier une exigence du projet au profit d'une autre. Comment le flux de problèmes à résoudre est-il géré par les chefs de projet étudiés?

L'enquête montre que ces derniers s'appuient en premier lieu sur une expérience partagée, à l'extension de laquelle ils participent activement. Dans l'hypothèse où cette expérience partagée ne suffirait pas, ils engagent un débat avec leurs collaborateurs (les salariés de leur entité affectés à leur projet) et sollicitent en parallèle l'appui des cadres qui travaillent au siège de leur entreprise. Mais c'est aux chefs de projet qu'il revient de «trancher», en faisant le cas échéant des paris sur la suite des événements. Ils peuvent pour cela compter sur leur «sens des réalités», c'est-à-dire sur leur «sens du possible et de l'impossible dans un contexte

(31) Entretien du 21 juillet 2010.

(32) Les projets d'investissement prévoient un montant affecté au poste «aléas». Le montant dégagé par Alban vient s'ajouter au montant affecté à ce poste. donné», leur «intuition de la marge de manœuvre » (BERLIN, 2011, p. 60) ${ }^{(33)}$.

\section{Prendre appui sur l'expérience collective et contribuer à son extension}

Quand les chefs de projet affrontent un problème, ils ne disposent ni du temps ni des ressources financières nécessaires à un traitement exhaustif de ses données; si le problème est complexe, cette exhaustivité est de toute façon impossible en soi.

Dans ces conditions, quelle que soit la nature ou l'importance du problème, la première chose qu'ils font pour y remédier est de s'appuyer sur les précédents. Ils se demandent s'ils ont déjà rencontré un problème comparable sur leurs projets antérieurs ou, à défaut, s'ils connaissent un chef de projet dont ce serait le cas; s'ils n'en connaissent pas, ils sollicitent leur hiérarchie pour une éventuelle mise en contact. S'il existe une solution au problème reconnue comme satisfaisante dans leur entreprise, les chefs de projet s'y réfèrent. Dans les sociétés observées, la procédure prescrit l'échange d'expériences entre chefs de projet. Les modalités de cet échange varient selon la société et la catégorie de chef de projet.

Les chefs de projet de l'entreprise de construction qui interviennent au stade de l'exécution des travaux (IRA) doivent renseigner ce qu'ils appellent une "fiche REX [retour d'expériences]» lorsqu'ils se heurtent à un problème. Ces fiches sont destinées à un service spécialisé, au siège, qui les classe par type de problème et tente d'en cerner les raisons. Quand c'est possible, ce service propose une solution. Autrement dit, il bâtit un répertoire de cas, de solutions éprouvées à des problèmes récurrents dont les causes ont pu être identifiées. Ce répertoire figure dans une base de données informatique que les chefs de projet consultent lorsqu'ils font face à un problème : ils cherchent en premier lieu «une solution REX sur le serveur» (IRA, 40 ans $\left.{ }^{(34)}\right)$. Si les chefs de projet ne sont pas tenus d'échanger leurs expériences lors de réunions formelles dédiées, les entretiens individuels avec leur hiérachie ainsi que leurs discussions informelles leur permettent également d'accéder utilement à ce que font leurs pairs puisque la base de données n'englobe pas l'intégralité des problèmes connus. En effet, plusieurs mois peuvent s'écouler entre le moment où un problème est décelé et celui où la solution apparaît éventuellement dans la base. De surcroît, c'est au chef de projet qu'il revient de juger si son problème mérite de faire l'objet d'une «fiche $R E X »$.

(33) Isaiah BERLIN (2011) définit le «sens des réalités» comme «le sens du possible et de l'impossible dans un contexte donné, le choix des moyens adaptés aux circonstances, l'intuition de la marge de manœuvre, le tout sans être capable d'expliquer pourquoi et comment on le sait, ni même toujours ce qu'on sait exactement» (p. 60).

(34) Entretien du 17 juin 2010. 
Pour les chefs de projet de la société exploitante, les modalités d'échange des expériences sont analogues, à ceci près que leur répertoire de cas ne figure dans aucune base de données : ce répertoire «s'incarne» dans la personne du directeur adjoint des investissements. Destinataire des rapports trimestriels dans lesquels les chefs de projet font état des problèmes qu'ils rencontrent dans leurs projets, ce dernier peut en effet les classer. Ensuite, en s'appuyant sur sa propre expérience et sur «l'histoire» des projets archivés, il identifie des solutions opératoires pour les difficultés dont il aura pu circonscrire les causes. Les chefs de projet de la société exploitante forment un groupe de taille réduite et le directeur adjoint des investissements, qui se déplace régulièrement dans leurs entités, est en lien constant avec eux. Aussi, lorsqu'ils sont confrontés à un problème, les chefs de projet sollicitent directement ce directeur, en espérant qu'il leur proposera une solution déjà expérimentée ailleurs ou qu'il les mettra en relation avec un collègue dont le projet présente des similitudes avec le leur. En outre, les «Journées annuelles», organisées par ce directeur, ont aussi vocation à mettre en relation les chefs de projet, qui échangent alors expériences et coordonnées.

Les chefs de projet de la société de déchets et ceux de l'entreprise de construction qui travaillent à la conception (IRP) ne renseignent aucune fiche qui serait destinée à un service spécialisé. Par contre, la procédure, dans leur entreprise, prescrit la tenue régulière de réunions d'échange d'expériences, encadrées par leur hiérarchie et dont le but est également de bâtir un répertoire de cas. Chacune fait l'objet d'un compte rendu qui figure dans une base de données, que ces chefs de projet consultent pour résoudre leur problème, mais aussi pour échanger de manière plus approfondie avec le collègue qui l'a déjà rencontré. L'un d'entre eux explique :

"On se réunit mensuellement: ce qui est arrivé ici chez l'un, arrivera ailleurs chez l'autre. [...] Quand il faut déplacer une espèce protégée, un avis du Conseil national de protection de la nature est requis; un chef de projet [déchets] a inauguré ça et il a fait un retour; on sait comment ne pas se rater.»

(Victor, 36 ans, CPD, société de déchets ${ }^{(35)}$ ).

Via l'échange (formel ou informel) d'expériences avec leurs homologues, les entrevues avec une hiérarchie disposant d'une vue sur l'ensemble des projets et, pour certains d'entre eux, la consultation d'une base de données, les chefs de projet étoffent leur répertoire de cas personnels, et ainsi élargissent l'éventail des solutions pouvant s'offrir à eux lorsqu'ils font face à une difficulté. Si le problème auquel ils se heurtent ne s'approche que de loin d'un cas connu, ils ont au moins une idée de ce qui est et, par-là, de ce qui pourrait être. En tout état de cause, ils améliorent leur capacité à bien gérer un projet en accédant aux expériences de leurs pairs ( on sait comment ne pas se rater»).

\section{"Faire travailler toute l'entreprise"}

L'examen des précédents est loin de toujours suffire face à un problème. Les chefs de projet doivent pourtant le résoudre et prendre, à cet effet, la décision appropriée. Pour ce faire, ils engagent en premier lieu un débat avec leurs collaborateurs, au sein de leur entité. Les salariés de l'entité qui sont affectés à leur projet sont mobilisés sur un versant du projet (technique ou financier, principalement). Selon le versant sur lequel ils se situent, le sens de leur travail diffère. Pour schématiser, pour le technicien attaché à l'exigence de qualité technique, le coût paraîtrait presque secondaire, tandis que le collaborateur mobilisé sur le versant financier privilégie, lui, plutôt l'exigence du coût. Dans ces conditions, lorsque les chefs de projet sont confrontés à une difficulté, ils tentent d'abord de trouver le compromis qui leur éviterait de privilégier une exigence du projet au détriment d'une autre. Mais d'après nos données d'enquête, il est rare qu'un arbitrage puisse être évité.

Les chefs de projet étudiés occupent par ailleurs une position nodale dans leurs entreprises. La gestion des projets engage l'ensemble des domaines de compétence de chacune des trois sociétés (et ce, même si lesdits projets y correspondent à une activité marginale): "C'est toute l'entreprise qui travaille sur le projet » (IRP, 28 ans $\left.{ }^{(36)}\right)$. Un chef de projet, face à un problème qu'il ne sait pas résoudre seul, sollicite donc non seulement l'avis des salariés qui travaillent avec lui sur son projet, mais aussi, dans deux cas principaux, l'expertise des cadres des directions centrales de son entreprise (cadres techniques, financiers, acheteurs, commerciaux, juristes, etc.) : ou bien il ne sait pas intégrer dans son projet un enjeu qui relève de leur domaine (il a par exemple besoin d'un éclairage sur un point de réglementation); ou bien il doit «trancher» entre les exigences de son travail. L'arbitrage relevant de l'expertise de certains de ces cadres, il fait alors appel à eux pour s'assurer qu'un élément important ne lui échappe pas et qu'il a une bonne compréhension des différents aspects du problème. Cette sollicitation vient nourrir le débat qu'il engage parallèlement avec ses collaborateurs.

Toutefois, cette sollicitation ne va pas toujours de soi. En effet, rien n'y oblige formellement - pas même la procédure. Élément encore plus déterminant, les chefs de projets, ayant probablement du mal à abandonner complètement leur identité première de spécialiste, ont tous tendance à s'attribuer la partie du travail qui correspond à ce que l'un d'eux a désigné, lors de l'entretien, par l'expression 
«compétence-phare»(37). Celle-ci peut renvoyer aussi bien à la compétence qu'un chef de projet a acquise en formation initiale qu'à la compétence associée au poste qu'il a le plus longtemps occupé durant sa carrière : le contenu de la «compétencephare» des chefs de projet étudiés varie donc en fonction de leurs parcours professionnels (38).

Mobiliser sa «compétence-phare» signifie deux choses qui sont liées : le chef de projet s'acquitte lui-même des tâches qui relèvent de sa spécialité première; lorsqu'un arbitrage à rendre touche à cette spécialité, il ne sollicite aucune autre expertise que la sienne propre, se contentant de solliciter les cadres du siège de son entreprise détenant une compétence dont il ne dispose pas lui-même.

$\mathrm{Si}$ les chefs de projet peuvent avoir intérêt à mobiliser leur spécialité première pour conduire leur travail (ils gagnent du temps), il reste que ces initiatives peuvent poser problème aux cadres qui travaillent au siège de chacune des trois entreprises étudiées. Selon tous les cadres du siège interrogés dans cette enquête, un chef de projet qui ne dépasse pas son identité professionnelle initiale et qui engage fortement sa «compétence-phare» dans son travail délaisse nécessairement certaines composantes de son projet, s'empêchant du même coup d'adopter un regard global sur celui-ci. Corollaire, quand ce chef de projet devra arbitrer entre les exigences de son travail, il aura probablement tendance à être moins exigeant sur les aspects du projet qui lui sont les moins familiers - ou bien à privilégier ceux qu'il maîtrise le mieux ${ }^{(39)}$.

C'est d'ailleurs avec les cadres du siège détenant la même compétence que celle des chefs de projet que la tension est la plus vive. Les observations des «Journées chefs de projet d'investissement» l'ont révélée. Ces chefs de projet n'ayant pas d'expertise en matière juridique et réglementaire, ils sollicitent régulièrement celle des cadres de la direction juridique. En revanche, ils détiennent une expertise en matière technique ou en matière d'achats : ils sont

(37) Entretien du 12 juillet 2010. L'intéressé, ingénieur diplômé spécialisé en hydraulique, désignait la compétence qu'il avait acquise en école d'ingénieurs.

(38) Bien entendu, les illustrations abondent. Mentionnons le cas des chefs de projet de l'entreprise de construction qui ont débuté leur carrière comme dessinateurs. Les projets de conception (IRP) et de construction (IRA) supposent une activité de dessin, relevant en principe de la responsabilité de l'ingénieur épaulant le chef de projet; sur les trois chefs de projet interrogés qui ont débuté leur carrière comme dessinateurs, tous affirment s'occuper, sur leurs projets, d'une partie du dessin; les autres chefs de projet rencontrés, qui ne sont donc pas dans ce cas, affirment au contraire que le dessin leur est parfaitement étranger (mais leur «compétence-phare» est différente, et ils l'engagent tout autant dans leur travail).

(39) Le directeur des ressources humaines de l'entreprise de construction - interrogé le 9 avril 2010 - considère ainsi que les chefs de projet de cette entreprise, qui sont globalement des techniciens de l'eau et de l'assainissement, ont «trop souvent » tendance à privilégier «la qualité technique» au détriment de «la rentabilité» du projet. le plus souvent recrutés dans ces fonctions. Lors des observations, les intervenants issus de la direction technique et de la direction des achats avertirent à maintes reprises les chefs de projet présents qu'une mauvaise réponse apportée à un problème touchant à leur domaine de compétence pouvait engendrer de graves difficultés. Le cadre de la direction des achats a conclu son intervention en les intimant de le solliciter «au moindre problème»:

«Au moindre problème, appelez! Mais ne faites pas à notre place.... Que chacun reste dans sa chapelle! Sinon, on ne s'en sort pas.»

(Cadre des achats, société exploitante $\left.{ }^{(40)}\right)$.

«La technique, c'est notre métier; le vôtre, c'est chef de projet! Donc, en cas de problème, informez systématiquement la direction technique.»

(Cadre technique, société exploitante ${ }^{(41)}$ ).

Ces cadres veulent contrôler les dimensions des projets sur lesquelles ils sont compétents ( $l a$ technique, c'est notre métier»; "que chacun reste dans sa chapelle!»). Leurs propos témoignent d'un phénomène qui s'apparente à une «lutte de juridiction» (АВBOTT, 2003). Un chef de projet qui empièterait sur le territoire du cadre des achats au motif qu'il serait un ancien acheteur nuirait à la qualité de son propre travail, à double titre : le cadre de la direction des achats étant le véritable expert en matière d'achat, son éclairage est indispensable au chef de projet, dès lors que celui-ci doit prendre une décision qui touche plus ou moins directement à ce domaine; si le chef de projet regarde son projet non par le seul prisme de sa spécialité mais dans sa globalité, alors il a de meilleures chances de faire les bons choix et d'atteindre sa cible. Si l'éclairage de l'expert du siège est réputé nécessaire à la prise de décisions équilibrées, le propos du cadre de la direction technique montre que l'expert n'entend pas rendre lui-même l'arbitrage : cet arbitrage est du ressort du chef de projet (et de sa hiérarchie). En revanche, ce cadre estime que, lorsque les arbitrages à rendre touchent au versant technique des projets, son expertise est trop peu sollicitée («informez systématiquement...»). Comme son homologue de la direction des achats, il considère qu'un chef de projet qui doit faire un choix à dimension technique ne peut qu'accroître ses chances de prendre la bonne décision en sollicitant l'avis de la direction technique.

$\mathrm{Au}$ fond, le propos de ces deux cadres du siège de la société exploitante peut s'entendre ainsi : les directions du siège ne sont pas dites «fonctions supports » pour rien; elles sont dotées de nombreux experts, dont le travail est notamment d'aider les entités territoriales, en particulier lorsque des projets y sont conduits.

(40) Observation du 13 octobre 2011.

(41) Idem. 


\section{Savoir "trancher"}

Nous allons envisager dans cette dernière section une situation que les chefs de projet de l'entreprise de construction qui sont chargés de la réalisation de travaux (IRA) rencontrent assez fréquemment : les études précédant l'engagement des travaux ne sont pas achevées à la date prévue dans le plan de coordination, et la question se pose de savoir s'il faut attendre leur achèvement ou s'il convient de démarrer immédiatement les travaux pour ne pas risquer des pénalités de retard - le montant de ces pénalités, défini contractuellement, est connu ex ante. Question complexe, car touchant à l'imbrication entre les exigences de délai, de coût et de qualité technique. Les études permettent de connaître les caractéristiques du site sur lequel les travaux seront réalisés; si ces caractéristiques sont insuffisamment connues au moment où les travaux débutent, alors les difficultés que les études auraient pu permettre d'éviter ou d'anticiper ${ }^{(42)}$ ne seront révélées qu'en cours de chantier. Elles obligeront donc à procéder à divers ajustements sur le chantier, coûteux en euxmêmes et plus ou moins longs à mettre en œuvre. Par exemple, tel matériau, finalement inadapté, doit être remplacé par tel autre; l'installation d'un équipement nécessite l'intervention d'un prestataire supplémentaire, qui aurait pu être évitée si tel problème avait été identifié plus tôt. Toutes sortes de cas sont possibles. Concrètement, chaque fois que ces chefs de projet sont confrontés à un retard dans la phase des études, ils essaient d'estimer le surcoût associé aux éventuels ajustements ultérieurs ainsi que leur durée, et procèdent par comparaison avec le montant des pénalités par jour de retard. Ils sollicitent divers avis (ceux de leurs collaborateurs et ceux des experts du siège) avant de décider, sous le contrôle de leur hiérarchie, de débuter ou non les travaux avant l'achèvement des études.

Le retard initial pris lors de la phase des études est la plupart du temps peu important. Dans ce cas de figure, l'expérience montre que le coût occasionné par les pénalités de retard est plus élevé que celui engendré par la décision de débuter les travaux à la date prévue. Autrement dit, quand le retard initial n'est pas très important, les chefs de projet choisissent généralement de faire débuter les travaux sans disposer de toutes les garanties apportées par les études. Bien sûr, plus le retard initial est important et plus ce choix est risqué : le coût d'adaptation de la construction en cours de chantier pourra non seulement être élevé en lui-même, mais cette adaptation pourra aussi être longue au point... d'empêcher la tenue du délai. Quand le retard est important, le risque est d'accroître le coût «technique»- le coût lié aux ajustements en cours de

(42) Les études ne permettent évidemment pas de tout prévoir. Même lorsqu'elles sont achevées, l'imprévu peut toujours survenir en cours de chantier. chantier, comme le rachat de telle ou telle pièce de l'usine - et de dépasser le délai ${ }^{(43)}$; le coût des pénalités de retard s'ajouterait au coût des ajustements et la rentabilité du projet s'en trouverait très significativement altérée.

Le chef de projet auquel nous nous intéressons spécifiquement ici - il s'agit de René - eut précisément à faire face durant sa carrière à un retard important, retard qu'il considérait comme "excessif». Mieux : ce caractère «excessif» du retard pris lors de la phase des études était inédit de son point de vue, ce qui explique pourquoi il estima crucial d'engager un débat avec les contributeurs de son entité (un acheteur et un ingénieur d'étude). De son propre aveu, les «discussions» avec ces derniers furent d'ailleurs «tendues». René sollicita aussi diverses expertises, au siège de son entreprise (technique, juridique et contractuelle, notamment). En définitive, après avoir examiné les solutions mises au point pour les cas de retard plus usuels, il fit le choix de respecter à la lettre le calendrier du projet : il décida que les travaux devaient débuter «le jour $J »$. René a donc «tranch[é]» :

«Il y a eu des discussions tendues avec l'acheteur et même avec l'ingénieur d'étude.... On ne sait pas ce qu'on va faire, mais je suis le porteur du projet, donc j'assume. ... Un débat s'instaure à trois, mais c'est à moi de décider. Pour tout, notamment quand c'est technique vs financier, la décision finale me revient; [...] je suis le chef de projet, le responsable, donc je tranche.»

(René, 47 ans, IRA, entreprise de construction (44)).

Dans les trois entreprises, les chefs de projet affirment ne rendre aucun arbitrage sans en avoir débattu avec les contributeurs de leur entité. Mais la décision relève de leur responsabilité («la décision finale me revient»), même si elle devra être validée par leur hiérarchie. Dès lors, s'ils demandent l'avis de leurs collaborateurs, ce n'est pas seulement pour accroître la probabilité que la décision prise soit opératoire; c'est aussi pour faciliter leur compréhension de la décision et leur permettre de prendre les dispositions idoines.

Il s'avère que, sans disposer formellement d'une autorité hiérarchique, tous les chefs de projet étudiés sont habilités à définir la finalité que le travail de leurs collaborateurs doit poursuivre dans le cadre du projet et la façon dont il faut en envisager la conduite. Voici ce qu'en dit par exemple Victor, un chef de projet de la société de déchets :

«Le but, c'est trouver la solution la plus équilibrée, pas faire le plus beau projet technique! Je n'ai pas d'autorité sur les gens en région, mais... [Ils doivent

(43) Lorsque les problèmes qui apparaissent en cours de chantier obligent à des ajustements très longs et coûteux, la hiérarchie du chef de projet essaie généralement de renégocier les termes du contrat avec le client.

(44) Entretien du 6 septembre 2010. 
quand même faire ce que vous dites]... Je porte le projet, donc...»

(Victor, 36 ans, CPD, société de déchets $\left.{ }^{(45)}\right)$.

Les chefs de projet étudiés affirment tous rencontrer peu de difficultés pour obtenir de leurs collaborateurs qu'ils acceptent la «décision finale». Mais ce n'est pas tant leur «compétence relationnelle» (MILBURN, 2002) qui l'explique que la fonction qu'ils remplissent. Il semble bien en effet qu'obéir au chef de projet soit une obligation liée au travail des collaborateurs. En rapprochant les réflexions d'Everett C. Hughes sur les professions (à statut) du cadre de l'organisation et du projet, les données d'enquête peuvent se lire de cette façon : les employeurs «concèdent» implicitement aux chefs de projet une «liberté d'action»; si ces derniers ne «revendiquent» aucun «mandat»(46), ni à titre individuel, ni à titre collectif, ils pensent tous qu'il leur revient bien de «définir les comportements » de leurs collaborateurs «à l'égard de tout ce qui touche » au projet (Hughes, 1996, pp. 99-100, p. 108). Et, de fait, rien ne le leur interdit. Par leur fonction («porter le projet»), quelles que soient par ailleurs leurs qualités individuelles, ils sont légitimes pour «indiquer ce qui est bon » pour le projet à leurs collaborateurs (p. 100). Si ces derniers s'y retrouvent, c'est sans doute aussi parce qu'obéir protège en cas d'erreur susceptible de compromettre la viabilité du projet voire de nuire à l'entreprise.

Mais le cas de René livre encore une autre clef de compréhension du travail du chef de projet. Reprenons le fil de l'histoire :

«J'ai fait un choix à un moment: commencer les travaux le jour J. On a fait des choix de travaux sans savoir où allaient passer les derniers tuyaux, les équipements, si c'étaient ceux-là.... On a pris des options ! Certaines, payantes, d'autres.... L'acheteur a été mécontent (ça lui a coûté plus cher) ... - on n'a pas fait n'importe quoi non plus, on a tapé au milieu et on a repris une route à peu près convergente... Si je m'étais complètement trompé, c'était fini...»

(René, 47 ans, IRA, entreprise de construction ${ }^{(47)}$ ).

La justesse de son choix était incertaine. En effet, chaque terrain sur lequel une usine est implantée est singulier; or les études réalisées sur le terrain de son usine étaient largement incomplètes. Lorsque René fit le choix de "commencer les travaux le jour $J$ », il savait qu'il devrait sans doute adapter la construction à ce qu'il découvrirait en cours de chantier et qu'il devrait prendre «des options». Mais, compte tenu de l'importance inédite du retard, il ne pouvait pas savoir si cette adaptation n'allait pas se révéler

(45) Entretien du 16 novembre 2010.

(46) Le «mandat» décrit un pouvoir de définir la manière dont l'activité doit s'exercer, mais aussi de «définir les comportements que devraient adopter les autres personnes à l'égard de tout ce qui touche» à l'objet de cette activité (Hughes, [1996], p. 99).

(47) Entretien du 6 septembre 2010. très longue et coûteuse (peut-être allait-il falloir remplacer des équipements après les avoir installés, par exemple). Il fit le pari que les ajustements seraient mineurs, dès l'instant où il serait prudent («on a tapé au milieu»). Si l'acheteur fut «mécontent », c'est parce que le choix de René le conduisit à acheter des pièces qu'il fallut ensuite remplacer par d'autres ("ça lui a coûté plus cher»). Cependant, au final, ce choix se révéla judicieux : le délai fut tenu et le surcoût lié aux ajustements fut relativement faible. Ainsi, René misa que ce surcoût serait moindre que le coût lié au dépassement du délai (pénalités de retard) et les faits lui donnèrent raison.

Ce cas est significatif du caractère conjecturel de la gestion de projet. Il prouve que l'exercice de ce métier «porte», en partie au moins, «sur une réalité qui échappe inévitablement à toute maitrise systématique » (CHАмРу, 2009, p. 84) et, corollaire, qu'il mobilise des savoirs qui ne sont pas complètement «formalisables» (CHAmpy, 2011). Les chefs de projet étudiés disent justement que, bien souvent, ils «sentent » à défaut de savoir; tous se fient, à un moment ou à un autre, à leur intuition (48). La probabilité que l'intuition se révèle juste est d'autant plus grande qu'elle est «lestée par l'expérience» (Champy, 2009, p. 85). Les chefs de projet s'appuient sur leur expérience en matière de conduite de projet et sur celle de leurs pairs - leur employeur organisant la circulation de ces expériences, ainsi qu'on l'a vu. Mais pas seulement.

Un autre élément leste l'intuition des chefs de projet étudiés, que l'on désignera sous le terme de «compétence opératoire», en référence aux travaux de Masahiko Aoki (Piotet, 1992 [selon M. Aoki]). Ce caractère opératoire décrit un surcroît de compétence qu'un travailleur acquiert par le fait de changer de poste dans son organisation; à mesure qu'il occupe des postes différents, il devient capable d'appréhender la globalité de son organisation; il acquiert une connaissance intime du procès de production en son sein, qui le sert dans son travail. Tous les chefs de projet étudiés possèdent ce type de compétence. Mais, dans leur cas, le caractère opératoire s'entend à l'échelle du secteur de l'environnement. À une exception près, aucun n'a débuté sa carrière comme chef de projet ${ }^{(49)}$. S'ils ont presque tous travaillé dans des domaines divers (exploitation, études, achats, conseil aux collectivités, administration, etc.), il est remarquable que leurs parcours soient en même temps circonscrits à ce secteur. Ils en connaissent intimement les arcanes. Ce sont d'abord des spécialistes de l'environnement et cette spécialité est nécessairement un recours quand il leur faut se fier à leur intuition.

(48) Le mot feeling revient régulièrement dans les entretiens. (49) L'exception concerne un ingénieur diplômé d'une école spécialisée dans l'environnement (voir encadré 2). 
Cet article ouvre la boîte noire du contenu du travail de chef de projet. Nous avons identifié à cet égard trois contributions essentielles à la réussite d'un projet, qui requièrent la constitution et la mobilisation d'appuis cognitifs et techniques spécifiques. Si la définition des éléments constitutifs de la cible à atteindre (coût, délais, spécifications techniques) échappe aux chefs de projet étudiés, c'est bien à ces «chefs d'orchestre», pour reprendre la métaphore bien connue, qu'il revient de bâtir le plan de coordination qui fera office de partition tout au long du projet. La difficulté de l'exercice tient à la nécessité d'articuler entre elles les exigences propres aux contributeurs directs et aux acteurs parties prenantes de la filière globale dans laquelle s'insère le projet, mais aussi à celle de jongler en permanence entre les visions macroscopique (vision globale) et microscopique (détails importants) de ce dernier. L'élaboration du plan de coordination permet par la suite d'exercer une surveillance sur un certain nombre de tâches qualifiées de critiques. Forts de cette vue globale du projet, dont ils sont les seuls à maîtriser tous les tenants et les aboutissants, les chefs de projet remplissent également une fonction de nature politique, au sens où ils «portent» le projet : ils l'incarnent et s'en font le porte-parole au cours des nombreuses réunions auxquelles ils participent.

La seconde contribution renvoie à une posture, la vigilance, qui permet de contenir les risques de dérapage inhérents à la mobilisation d'acteurs hétérogènes, entretenant des liens plus ou moins forts au projet et à ses buts, intervenant dans un milieu à l'irréductible singularité. Les chefs de projet étudiés ne se contentent pas d'être à l'affût des «grains de sable» susceptibles de mettre à mal l'ordonnancement précédemment élaboré; leur vigilance est réflexive, en ceci qu'elle s'ajuste aux éléments dont l'expérience a montré l'importance. Elle est aussi proactive au sens où elle s'accompagne d'un effort de constitution de «réserves», notamment financières, permettant d'absorber certains aléas.

Si le «chef» incarne le projet et veille à son bon déroulement, il est aussi celui qui règle les problèmes. L'analyse montre que, pour s'acquitter de cette troisième contribution, les chefs de projet étudiés peuvent d'abord compter sur une expérience collective, un capital accumulé de problèmes et de solutions, bien commun dont ils bénéficient et à l'extension duquel ils sont explicitement appelés à contribuer. Leur analyse bénéficie aussi de l'éclairage apporté par les contributeurs internes, qui sont porteurs, chacun à leur niveau, d'un point de vue singulier sur le projet. Il reste qu'il leur revient d'arbitrer entre d'éventuelles exigences contradictoires et de faire face à l'incertain, dans une solitude relative, tempérée par la présence d'arrière-plan de la hiérarchie : au terme d'une délibération conduite avec les collaborateurs concernés, s'appuyant sur leur vue globale du projet, forts d'un sens du possible nourri de leurs précédents projets et de leur parcours professionnel, ils décident et même, ils prennent «des options» en espérant qu'elles se révèleront «payantes». On retouve ici une autre dimension politique du travail de gestion de projet : le chef de projet est aussi celui qui «tranche».

Le portrait des chefs de projet qu'esquisse notre analyse est donc celui de professionnels qui affrontent une épreuve incertaine et qui puisent à de multiples expériences pour porter un jugement équilibré sur les problèmes auxquels ils font face. Cette pratique prudentielle s'exerce dans le contexte particulier de la subordination salariée. La hiérarchie des chefs de projet se réserve la possibilité d'imposer ses arbitrages au regard des contraintes de l'entreprise (la rentabilité des investissements, par exemple) et d'un sens du possible plus étendu (prise en compte d'autres dimensions) et plus profond (accumulation supplémentaire de précédents). En contrepartie, on l'a vu, ces chefs de projet interviennent dans un contexte «environné», qui soulage de multiples façons la charge cognitive de pilotage du projet et d'arbitrage. Ils sont d'ailleurs invités à faire partie d'une communauté de pratiques qui, si elle ne dispose pas de l'autonomie d'une profession protégée, habilite néanmoins ces praticiens à se construire une identité collective et un socle partagé de préoccupations et d'expériences. Notre analyse invite en fin de compte à détacher les pratiques prudentielles d'une association peut-être trop étroite avec les professions protégées. La prudence apparaît moins ici comme une qualité personnelle requérant l'autonomie dans l'exercice d'une activité que sous la forme d'une qualité distribuée dans un réseau étroit de dispositifs organisationnels : les chefs de projet sont sans cesse invités à produire des traces de leur activité (comptes rendus, fiches d'expérience, etc.), ce qui les invite à la réflexivité et les expose à la vigilance critique de leurs pairs et de leur supérieurs ; les fonctions supports nourrissent le jugement qui précède l'arbitrage et les prémunissent contre certains biais; leur hiérarchie veille à faire circuler les références, à «globaliser» leur appréhension du projet, en même temps qu'elle valide leurs principales options. La prudence est donc distribuée au sein de ces obligations de forme, de l'intervention des fonctions supports et de ces liens à la fois hiérarchiques et fonctionnels avec des pairs plus expérimentés. 


\section{Bibliographie}

Аввотт A. (2003), «Écologies liées : à propos du système des professions », in Menger P.-M. (dir.), Les professions et leurs sociologies. Modèles théoriques, catégorisations, évolutions, actes du colloque de la Société française de sociologie, Paris octobre 1999, Paris, Éditions de la Maison des sciences de l'homme, pp. 29-50.

BERLIN I. (2011), Le sens des réalités, Paris, Les Belles Lettres.

Bouffartigue P. (2001), Les cadres. Fin d'une figure sociale, Paris, La Dispute.

BRION S. (2005), «Le concept de vigilance. Une réponse au dilemme entre rapidité et fiabilité des projets », Revue française de gestion, vol. 31, no 3, pp. 105-128.

Снамpy F. (2011), Nouvelle théorie sociologique des professions, Paris, Presses universitaires de France.

Champy F. (2009), La sociologie des professions, Paris, Presses universitaires de France.

Cochoy F. (2002), «Une petite histoire du client, ou la progressive normalisation du marché et de l'organisation », Sociologie du travail, vol. 44, no 3, pp. 357-380.

Duval R. (1990), Temps et vigilance, Paris, Vrin.

Garel G. (2011), Le management de projet, Paris, La Découverte.

Granjon M.-C. (1999), «La prudence d'Aristote : histoire et pérégrinations d'un concept », Revue française de science politique, vol. 49, n 1 , pp. 137-146.

Hughes E. C. (1996), Le regard sociologique. Essais choisis, Paris, École des hautes études en sciences sociales.
Karvar A., Rouban L. (dir.) (2004), Les cadres au travail. Les nouvelles règles du jeu, Paris, La Découverte.

LeFEBVRE P. (2003), L'invention de la grande entreprise. Travail, hiérarchie, marché. France, fin XVIIIe-début $X X^{e}$ siècle, Paris, Presses universitaires de France.

Livian Y.-F. (dir.) (2004), «Ce que font les cadres. Actes de la journée du 8 décembre 2003 organisée par l'IAE de Lyon », Les cahiers du GDR Cadres, n 6.

Merchiers J., Pharo P. (1990), «Compétence et connaissance expertes. Propriétés publiques et cognitives-pratiques», Sociétés contemporaines, vol. 4, no 4 , pp. 89-108.

Midler C. (1993), «Le responsable de projet, portrait d'un rôle d'influence», Gestion 2000, vol. 9, no 2, pp. 123-147.

Milburn P. (2002), «La compétence relationnelle: maîtrise de l'interaction et légitimité professionnelle. Avocats et médiateurs », Revue française de sociologie, vol. 43, no 1, pp. 47-72.

Piotet F. (1992), «Coopération et contraintes. À propos des modèles d'Aoki », Revue française de sociologie, vol. 33, no 4, pp. 591-607.

Segrestin D. (2004), Les chantiers du manager, Paris, Armand Colin.

VINCK D. (dir.) (1999), Ingénieurs au quotidien: ethnographie de l'activité de conception et d'innovation, Grenoble, Presses universitaires de Grenoble. 


\section{La construction du matériau empirique}

Le matériau est constitué de trente-cinq entretiens auprès de trente individus parmi lesquels on compte vingt-deux chefs de projet : dix chefs de projet investissement (CPI), quatre ingénieurs responsable projet (IRP), quatre ingénieurs responsable d'affaire (IRA) et quatre chefs de projet déchets (CPD). Les cinq autres entretiens eurent lieu avec un directeur des ressources humaines (DRH) du groupe et le DRH de chaque filiale; deux cadres du service «Processus et Organisation» de l'entreprise de construction; le directeur des investissements et son adjoint (société exploitante).

Les entretiens avec les chefs de projet ont été enregistrés et la transcription adressée à chacun pour relecture ${ }^{(1)}$. Peu directifs, ils furent centrés sur leur parcours, le contenu de leur travail et le contexte de son exercice. Il fut spécialement demandé à chacun de détailler ce contenu en prenant appui sur le projet sur lequel il travaillait au moment de l'entretien, mais aussi de décrire les erreurs qu'il avait pu commettre, la manière dont il tentait de résoudre les problèmes rencontrés et ce qu'il avait fait (ou pensait qu'il eût fallu faire) face à ceux qu'il avait connus précédemment.

En dehors des CPD de la société de déchets, les individus interrogés furent définis conjointement avec la DRH du groupe et celle des deux autres filiales. La DRH de la société de déchets s'étant de fait soustraite à l'enquête (2), les CPD interrogés furent ceux qui avaient par ailleurs un lien avec l'école partenaire du groupe. La DRH de la société exploitante nous a orientés vers le directeur des investissements, qui transmit la liste des CPI et présenta le profil de ceux dont il suggérait l'interview; la variété de ces profils (âge, niveau de diplôme, etc.) justifia de les interroger - tous en avaient accepté le principe. Enfin, la DRH de l'entreprise de construction transmit une liste d'IRP/IRA ne comprenant d'autres données que le genre et l'ancienneté dans la société. Les (rares) IRP/IRA ayant accepté un entretien ont donc été choisis selon ces critères. Afin de saisir le contexte dans lequel ils évoluaient, il fut décidé d'essayer de les recruter dans la même entité : parmi eux, cinq travaillent dans une entité où les projets sont de petite taille.

Treize observations furent réalisées concomitamment aux entretiens : cinq réunions de chantier (société exploitante, entreprise de construction); une formation à la gestion de projet (société exploitante); deux «Journées CPI» (société exploitante); cinq interventions de chefs de projet (IRP et CPD) auprès d'élèves-ingénieurs. Plus de la moitié des chefs de projet (treize) ont ainsi été vus plusieurs fois, dans des contextes différents.

De nombreuses informations furent collectées en situation d'enquête : procès-verbaux de réunion, données techniques, contractuelles, financières du projet, organisation de l'entité, etc. Au niveau des directions des trois sociétés et du groupe, si les fiches de poste ont par exemple été obtenues plutôt facilement, d'autres éléments restèrent malheureusement inaccessibles, comme les propriétés sociographiques générales de la population des chefs de projet ou les organigrammes.

(1) Les enregistrements durent plus de trois heures en moyenne. Les entretiens ont été réalisés sur leurs lieux de travail. Sept chefs de projet furent rencontrés pendant une journée entière ( 9 heures -17 heures). Cinq d'entre eux furent observés lors d'une réunion de chantier qu'ils animaient, le matin; l'entretien eut lieu l'après-midi. La journée passée avec les deux autres fut consacrée à l'entretien ainsi qu'à la visite des locaux de leur entité, d'une usine située à proximité et du chantier sur lequel ils travaillaient.

(2) Les raisons ayant conduit la DRH de cette société à ne pas participer à l'enquête nous sont inconnues. Toutes nos relances sont restées sans suite, sans qu'aucune raison nous ait jamais été donnée. 